\title{
Pattern reversal evoked visual potential in the diagnosis of multiple sclerosis
}

\author{
W. B. MAT THEW S ${ }^{1}$, D. G. SMALL ${ }^{2}$, MAR I A N SMALL, A N D \\ ER I K A POUNT NEY
}

From the University Department of Clinical Neurology, Churchill Hospital, Oxford

SUMMARY The pattern reversal evoked visual potential (VEP) was recorded in 37 normal subjects and in 186 patients in whom a diagnosis of multiple sclerosis (MS) was established or suspected. Taking the upper limit of normal as the mean $+2.5 \mathrm{SD}(111 \mathrm{~ms})$, prolonged latency was found in $75 \%$ of definite cases of MS, $58 \%$ of probable cases, and $38 \%$ of possible cases. A smaller number of patients without prolonged latency had abnormal asymmetry of latency or low amplitude potentials. In patients with a single acute episode of neurological disease resembling MS the incidence of abnormal VEP was very low. In patients examined within three months of an episode of retrobulbar neuritis (RBN), latency was prolonged in $81 \%$ of affected eyes, a similar proportion being found in patients with a more remote history of RBN. The importance of establishing the normal for every laboratory engaged on this investigation is emphasised. Prolonged latency of the VEP is common in established MS but has not yet been shown to be a sensitive diagnostic test of the early case.

The latency of the main positive deflection of the pattern reversal evoked potential (VEP) recorded from the scalp was found by Halliday et al. (1972) to be prolonged in optic neuritis, and to be persistently abnormal after recovery of visual acuity. The use of the technique was then logically extended to the demonstration of subclinical involvement of the visual system in patients with established or suspected multiple sclerosis (MS), thus providing objective evidence of multiple lesions (Halliday et al., 1973). Many centres are now employing this method and it is necessary to examine its value and limitations.

\section{Method}

The stimulus was a black and white checkerboard pattern projected from a slide on to a screen in front of the subject. The pattern subtended an angle of $23^{\circ}$ at the subject's eye at a distance of $1.6 \mathrm{~m}$, each square subtending an angle of $28^{\prime}$. The luminance of the individual white squares was

\footnotetext{
'Address for reprint requests: Professor W. B. Matthews, University Department of Clinical Neurology, Churchill Hospital, Oxford England.

'Present address: The National Hospital for Nervous Diseases, Queen Square, London WCIN 3BG.

Accepted 11 July 1977
}

$256 \mathrm{~cd} \mathrm{~m}^{-2}$ and of the black squares $38 \mathrm{~cd} \mathrm{~m}^{-2}$. A mirror directing the light from the slide to the screen rotated through a small angle causing the pattern to move horizontally the width of one square twice a second. Each rotatory movement of the mirror was completed in less than $5 \mathrm{~ms}$. Scalp potentials were recorded via conventional silver/silver chloride disc electrodes, the active one being placed $50 \mathrm{~mm}$ above the inion in the midline with a mid-frontal reference $(\mathrm{Fz}, 10-20$ system), and after amplification (bandwidth $-3 \mathrm{db}$ at 0.16 and $250 \mathrm{~Hz}$ ) were summated in a digital averager. The averager sweep was triggered at the onset of pattern displacement and lasted 200 ms with a sampling rate of 1 point $/ \mathrm{ms}$ : 256 responses were summed from each eye tested separately. The subject reclined on a couch with the head raised and was instructed to fixate a small red light in the centre of the screen, the rest of the room being darkened.

\section{Results}

Thirty-seven normal subjects with corrected visual acuity of $6 / 6$ or better were examined. The age range was from 17 to 56 years. The mean latency of the major positive potential of the VEP (P100) in the 74 eyes was $100.5 \mathrm{~ms}$ (range $90-113 \mathrm{~ms}$, SD 
4.37). The mean latency in the seven subjects over 40 years of age was not significantly longer, being $102 \mathrm{~ms}$. In individual subjects the latency from the two eyes did not differ by more than $6 \mathrm{~ms}$. The amplitude of the potential ranged from 4-33 $\mu \mathrm{V}$ with a markedly skewed distribution. An abnormal response was taken as absence of a measurable potential; or a latency greater than the normal mean $+2.5 \mathrm{SD}(111 \mathrm{~ms})$; or a difference in latency between the response from the two eyes greater than $7 \mathrm{~ms}$; or an amplitude of less than $4 \mu \mathrm{V}$.

Between May 1974 and August 1976 we examined 186 patients in whom the diagnosis of MS was regarded as established or had been reasonably entertained. Thirty of these patients had either sustained an attack of retrobulbar neuritis (RBN) within the previous three months or had exclusively visual symptoms and are considered separately. Of the remaining 156,110 could be classified as definite, probable, or possible cases of MS according to the criteria of McAlpine et al. (1972). Two additional categories were necessary. Patients with a single episode of neurological symptoms, other than visual failure, of less than three months' duration, were classified as 'acute, not diagnosed' (AN). Of the 39 patients in this group, 14 had sensory symptoms that could readily be attributed to MS, eight had isolated diplopia, eight had symptoms of brain stem involvement, and three had subacute paraplegia. Four patients had localised sensorimotor symptoms, one had cerebellar ataxia, and one had undiagnosed remitting cerebral disease with bilateral extensor plantar reflexes. Patients with chronic undiagnosed neurological disease in whom MS had been considered but appeared improbable were classified as 'chronic, not diagnosed' (CN). This small category included progressive cerebellar ataxia but not progressive unexplained paraplegia without evidence of disease beyond the spinal cord, as this, by definition, falls into the possible MS group.

A number of patients were examined on two or more occasions but later results are included only from two patients in whom the course of the disease necessitated reclassification from possible to definite, and from one of these who, when in the definite category, developed RBN with subsequent changes in the VEP. Results of 159 examinations in these 156 patients are, therefore, presented. The distribution between the five categories is shown in Table 1.

Of the 30 patients with recent RBN or exclusively visual symptoms, 24 had sustained an observed attack of RBN within the three months preceding the examination, four of them within two weeks. In 19 of these, there were no other
Table 1 Diagnostic categories at time of investigation of 156 patients without recent retrobulbar neuritis or exclusively visual symptoms in whom multiple sclerosis was established or suspected. As explained in the text, one patient was investigated twice, and one three times, accounting for the total of 159

\begin{tabular}{lc}
\hline Category & Number \\
\hline Definite & 61 \\
Probable & 24 \\
Possible & 28 \\
AN & 39 \\
CN & 7 \\
Total & 159 \\
\hline
\end{tabular}

features of MS, but one was classified as a possible case, three as probable, and one as definite. In four patients without symptoms or signs of disseminated disease there was a past history of RBN, unilateral in one, and affecting both eyes in separate episodes in three. In the remaining two patients, the only complaint was of dimness of vision on exertion with no history of RBN, normal visual acuity, and no pallor of the optic discs.

The results of 159 examinations in the 156 patients without recent or exclusively visual symptoms are shown in Table 2, using prolonged latency or an absent response as the sole criterion of abnormality. It is clear that there is a diminishing frequency of abnormal results, descending from $75 \%$ in the definite cases to $58 \%$ in the probable group and $38 \%$ in the possible cases, with much lower figures in the two undiagnosed categories. Increasing certainty of diagnosis is also strongly associated with increasing abnormality of latency of response from stimulation of individual eyes (Figure).

In the definite group attention should be drawn to the two patients with a past history of RBN and pale discs but normal latency of the VEP. In both these patients the amplitude of the potential was abnormally low from both eyes. Abnormally low amplitude with normal latency was found in only one other patient in the whole series, also in the definite group. This patient, however, had no history of visual disorder, normal optic discs, and a latency actually below the normal range from both eyes. Abnormal asymmetry of latency, in the absence of delay, was not found in any patient with definite MS. The addition of the three patients with low amplitude potentials raises the percentage of patients with abnormalities from $75 \%$ to $80 \%$. The results of two examinations in one patient in this group are included. Before she had sustained an attack of RBN, VEPs were normal, but when recorded several months later, latency was pro- 
Table 2 Results of 159 recordings of VEP in 156 patients with established or suspected multiple sclerosis but without recent retrobulbar neuritis or exclusively visual symptoms, using prolonged latency as the only criterion of abnormality

\begin{tabular}{|c|c|c|c|c|c|c|c|c|}
\hline & & Definite & Probable & Possible & $A N$ & $C N$ & Total & $\%$ Delayed \\
\hline History of RBN & $\begin{array}{l}\text { Delayed } \\
\text { Normal }\end{array}$ & $\begin{array}{r}26 \\
2\end{array}$ & $\begin{array}{l}4 \\
2\end{array}$ & $\begin{array}{l}0 \\
2\end{array}$ & $\begin{array}{l}0 \\
0\end{array}$ & $\begin{array}{l}\mathbf{0} \\
0\end{array}$ & 36 & 83 \\
\hline No RBN, Disc pallor & $\begin{array}{l}\text { Delayed } \\
\text { Normal }\end{array}$ & $\begin{array}{l}9 \\
4\end{array}$ & $\begin{array}{l}3 \\
2\end{array}$ & $\begin{array}{l}2 \\
0\end{array}$ & $\begin{array}{l}0 \\
6\end{array}$ & $\begin{array}{l}0 \\
2\end{array}$ & 27 & 48 \\
\hline Nil visual & $\begin{array}{l}\text { Delayed } \\
\text { Normal }\end{array}$ & $\begin{array}{r}11 \\
9\end{array}$ & $\begin{array}{l}7 \\
6\end{array}$ & $\begin{array}{r}8 \\
16\end{array}$ & $\begin{array}{r}2 \\
31\end{array}$ & $\begin{array}{l}1 \\
5\end{array}$ & 96 & 30 \\
\hline $\begin{array}{l}\text { Total } \\
\% \text { Delayed }\end{array}$ & & $\begin{array}{l}61 \\
75\end{array}$ & $\begin{array}{l}24 \\
58\end{array}$ & $\begin{array}{l}28 \\
38\end{array}$ & $\begin{array}{r}39 \\
5\end{array}$ & $\begin{array}{l}7 \\
7\end{array}$ & 159 & \\
\hline
\end{tabular}

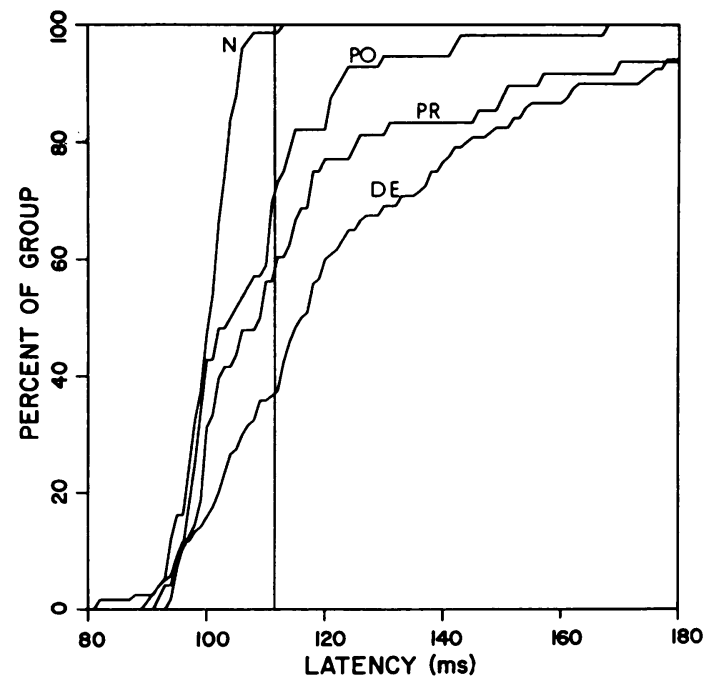

Figure Integrated histograms of latency of major positive peak of VEP (P100) for normal subjects and for possible $(P O)$, probable $(P R)$, and definite $(D E)$ categories of multiple sclerosis. Each histogram represents, at a given latency, the number of eyes with a VEP latency less than or equal to this value, expressed as a percentage of the total number of eyes in the group. Representing the data in this way emphasises the large change in the proportion of patients classified as abnormal which occurs for a small change in the upper limit of normal latency. The vertical line representing the latter is drawn at $111.5 \mathrm{~ms}$.

longed from the eye that had been affected. The severity of the disease in the 13 patients in whom no abnormality of VEP was found did not differ obviously from that in the remainder of this group. None had a history of RBN and optic disc pallor was thought to be present in only four.

In the probable group there were also two patients with a past history of $\mathrm{RBN}$ and pale discs but normal latency of the VEP, but in these patients the amplitude was normal. In no patient in this group were the latencies from the two eyes abnormally asymmetrical in the absence of delay, nor was abnormally low amplitude found as an isolated abnormality. These two features do not, therefore, contribute to the proportion of patients with abnormal VEP which remains at $58 \%$.

The possible group of 26 patients contains, by definition, nine patients with progressive paraplegia without evidence of disease beyond the spinal cord but where alternative diagnoses had been excluded. In four of these, latency from both eyes was prolonged and in a further three patients the VEP was not delayed but latency from the two eyes was abnormally asymmetrical. In no patient in the possible group was the amplitude below the normal range. The percentage abnormality in patients with progressive paraplegia was, therefore, $78 \%$. In the remaining 19 patients in this group, latency was prolonged in six, and in one further patient there was abnormal asymmetry without delay. The additional factor of asymmetry raises the proportion of abnormal results in the possible group from $38 \%$ to $50 \%$ but this increase is heavily weighted by the patients with progressive paraplegia. Two patients were examined when classified as possible cases and again when the disease was definite. In one, VEPs were normal when examined in both the possible and definite category, and only became abnormal after an attack of RBN. The other patient was the one already referred to as having asymmetrical latencies. When examined again after progression to the definite stage, prolonged latency was found from both eyes. The inclusion of two patients with a history of $\mathrm{RBN}$ in the possible group is due to the doubtful nature of the visual symptoms in one and of the disseminated symptoms in the other.

The incidence of abnormal latency in the un- 
diagnosed $\mathrm{AN}$ and $\mathrm{CN}$ groups was very low. In the acute group, the only two abnormal results were in one patient with sensory symptoms and one with paraplegia. Asymmetry of normal latency and reduction of amplitude did not occur. In the chronic $\mathrm{CN}$ group the only abnormality was a slight prolongation of latency from one eye in a patient with unexplained sensory symptoms but with corrected acuity reduced to $6 / 24$ by refractive error.

In the three diagnostic categories of multiple sclerosis $62 \%$ of patients had prolonged latency of VEP and, using the additional criteria of asymmetry of latency and low amplitude, this proportion can be raised to $68 \%$. In those with no history of RBN and normal optic discs, prolonged latency was found in $45 \%$ and some abnormality of VEP in $54 \%$. If the acute and chronic undiagnosed groups are included these proportions fall to $30 \%$ and $32 \%$ respectively. Pallor of an optic disc without a history of RBN was accompanied by delayed VEP in $70 \%$ of patients with MS but no abnormality of VEP was found in patients in the undiagnosed categories who were thought to have pale discs. Of patients with a past history of RBN, $83 \%$ had prolonged latency and $89 \%$ had some abnormality of VEP.

Many of the patients in the AN group had symptoms suggestive of a first attack of multiple sclerosis. An attempt was made to determine how many of these subsequently developed definite evidence of the disease. Follow-up has been brief and in no case has exceeded two years. Of these 39 patients eight could not be traced. Twenty-six were known not to have developed further symptoms by the summer of 1976 . In two patients the symptoms investigated were shown not to be due to MS, one developing a brain stem glioma and one mononeuritis multiplex. Two patients entered the diagnostic categories of MS. One paraplegic patient, in whom symptoms persisted, by definition entered the possible MS group. One patient who presented with sensory symptoms developed definite MS, including RBN. These two patients were the only cases in the AN group in whom abnormalities of VEP had been found.

The results in 28 patients with recent $R B N$ or exclusively visual symptoms resulting from RBN in the past are shown in Table 3. The general pattern is, as expected, for there to be prolonged latency to stimulation of the recently affected eye and normal latency from eyes not known to have been affected by RBN, but there were many exceptions. From six eyes subject to RBN within the previous three months, one as recently as two weeks before the investigation, latency was nor-
Table 3 Latency of VEP from 56 eyes in 24 patients with recent retrobulbar neuritis and four patients with exclusively visual symptoms resulting from past episodes of $R B N$

\begin{tabular}{lllll}
\hline & \multicolumn{4}{l}{ Latency of VEP } \\
\cline { 2 - 5 } & Abnormal & Normal & Total & $\%$ Abnormal \\
\hline Acute RBN & 22 & 6 & 28 & 79 \\
Past RBN & 9 & 1 & 10 & 90 \\
No RBN & 6 & 12 & 18 & 33 \\
Total & 37 & 19 & 56 & 66 \\
\hline
\end{tabular}

mal, despite acuity reduced to $6 / 24$ in two and $6 / 18$ in one at the time of the examination, while latency was prolonged from six eyes where there was no history of RBN. In this group latency was prolonged from nine out of 10 eyes affected by RBN between four months and eight years before the investigation. Abnormal asymmetry of latency as defined did not contribute to the number of abnormalities found, but in one patient with normal latency three months after RBN the amplitude of the VEP was abnormally low. The two young women whose only complaint was of dimness of vision on exertion are not included in this table, but abnormalities were found in both. One had prolonged latencies and the other, with normal latencies, had VEP of abnormally low amplitude. On brief follow-up, no further symptoms have developed. In the whole series there was no close relationship between abnormalities of latency and amplitude and visual acuity, it being commonplace to find grossly prolonged latency with normal acuity and, less frequently, marked reduction of acuity with normal latency.

\section{Discussion}

The mean latency of the normal pattern reversal evoked potential has varied considerably between different studies. Halliday et al. $(1972,1973)$ originally reported a value of $120.1 \mathrm{~ms}$ and later, with improved technique, $103.8 \mathrm{~ms}$. Asselman et al. (1975) found a considerably shorter value of 90.5 ms. Our mean of $100.5 \mathrm{~ms}$ lies between these figures. The differences are no doubt accounted for by minor variations in technique, but it must be emphasised that normal values must be established in every laboratory embarking on this investigation as a diagnostic test.

Different standards of normality have also been adopted, Halliday et al. (1973) regarding as abnormal values in excess of the mean $+2.5 \mathrm{SD}$, while Asselman et al. (1975) were more stringent and required mean +3 SD. Mastaglia et al. (1976) did 
not state their mean normal latency, but regarded as abnormal values in excess of mean $+2.5 \mathrm{SD}$ which they state to be $118 \mathrm{~ms}$, implying a mean value in excess of our value of $100.5 \mathrm{~ms}$. We accepted mean $+2.5 \mathrm{SD}$ as the limit of normality $(111 \mathrm{~ms})$. By this standard, latency from one normal eye out of 74 was $2 \mathrm{~ms}$ beyond the normal range, which is what would be expected on statistical grounds. If mean $+3 \mathrm{SD}(114 \mathrm{~ms})$ had been taken as the limit of normality, all the control values would be below this figure, but seven additional patients, three definite, one probable, and three possible, would have fallen within the normal range, as would both the patients with acute undiagnosed neurological symptoms in whom prolonged latencies were found. In the diagnostic categories of MS the proportion of patients with delayed VEP would be reduced to $55 \%$. The significance of slight delay in the latency of the VEP is obviously debatable, and the diagnostic value of a latency 1 or $2 \mathrm{~ms}$ beyond the normal range cannot be regarded as equivalent to increases of $50 \%$ or more often seen in patients with advanced MS. This point is emphasised by the Figure when the responses from individual eyes are considered. It can be seen that a small shift to right or left of the vertical line indicating 'normality' would have an important effect on the in ${ }^{+}$erpretation of the results.

It is certainly to be hoped that study of evoked potentials will throw light o nthe disease process in MS but we are concerned here with their use in diagnosis. All have agreed that in definite MS there is a high proportion of abnormalities of latency, 84\% (Asselman et al., 1975), 83\% (Mastaglia et al., 1976), 97\% (Halliday et al., 1973 ), and $75 \%$ in our larger series. In these patients the diagnosis has, however, already been established on other grounds. In all patients diagnosed as MS in the usual three grades of certainty our proportion of patients with delayed VEP was $62 \%$. Including abnormalities of symmetry of latency and abnormally low amplitude this figure can be raised to $68 \%$. These figures are close to the $67 \%$ of delayed VEP reported by Asselman et al., 1975. and higher than the $50 \%$ of Mastaglia et al., 1976, but much lower than the $96 \%$ found by Halliday et al. (1973).

It is in the possible cases or patients with a single undiagnosed episode of neurological disease that diagnostic assistance is required. In these patients the incidence of abnormal latency is much lower either because they do not have MS or because the technique does not detect the early case. Halliday et al. (1973) found prolonged latency in 11 out of 12 possible cases, but others have not approached this figure. Asselman et al. (1975) reported $21 \%$ abnormal results in the possible group, but numbers are small and this percentage implies only three patients with abnormal latency. Mastaglia et al. (1976) reported 33\% abnormal latency in possible cases in a rather larger group but again only comprising 12 patients with abnormalities. Our figure of $35 \%$ is comparable and, by taking into account abnormal asymmetry of latency, is increased to $50 \%$. However, before such results can be claimed to show that the technique is 'a sensitive test of early MS' (Asselman et al., 1975), it must be recalled that in the classification of McAlpine et al. (1972) used, with modifications, by all observers, the possible group includes chronic progressive paraplegia without other discoverable cause. Once spinal tumour is excluded, most such patients, if followed to necropsy, are found to have multiple sclerosis (Marshall, 1955). Of our nine patients in this category, seven had either prolonged or asymmetrical latencies, and Halliday et al. (1974) found abnormal latency in five of 13 patients with chronic progressive paraplegia of unknown cause that could be classified as possible MS. In the brief follow-up so far attained in our possible cases, two have progressed to the definite category. In one, VEPs were normal when in the possible category and also when the disease became definite, only becoming abnormal after an attack of RBN. In the other patient, latencies were asymmetrical but otherwise normal early in the disease but were prolonged in the definite stage.

Asselman et al. (1975) reported a high incidence of delayed VEP in patients probably corresponding to certain categories of those we have classed as 'acute, not diagnosed'. In particular, they found abnormalities in six of 13 cases of isolated brain stem lesions. In contrast, we found no abnormal results in 16 patients with either acute brain stem symptoms and signs or isolated diplopia. Halliday et al. (1973) similarly found normal results in six patients with brain stem lesions. In the whole group of 39 patients with acute isolated episodes of apparent central nervous system disease, only two abnormalities were found. The VEP was slightly delayed in one eye in the only two patients who are definitely known to have subsequently entered one of the diagnostic categories of MS. At first sight this appears an impressive result, but to establish the value of this technique in early diagnosis prolonged follow-up of this group of patients, many of whom have presented with one of the common modes of onset of MS, is essential. Follow-up is, however, difficult to achieve as young adults are highly mobile and, if feeling well, are 
reluctant to renew medical contacts. The possibility of causing unjustified alarm must also be considered. There is at present insufficient evidence on which to base a definite statement on the use of pattern reversal VEP in the diagnosis of the early case of MS.

Our results in patients with recent $\mathrm{RBN}$ are surprising in that latency was normal from six out of 28 eyes examined within three months. Halliday et al. (1972) found prolonged latency from all but one of the 18 eyes they examined, but more of their cases were seen in the acute stage with much reduced visual acuity. In these patients we also found prolonged latencies from one-third of eyes where there was no history of RBN. Because of this the contrast between the affected and unaffected eyes, so striking in the series of Halliday et al. (1972) was much less evident. In one patient normal latency was obtained from the eye in which a mild attack of RBN had occurred three weeks previously, while latency from the supposedly normal eye was prolonged. The two patients who complained of dim vision on exertion appeared to have Uhthoff's phenomenon and both had abnormal VEP. There is no other evidence that they have MS.

While we were unable to confirm the original report of very high incidence of prolonged latency of the pattern reversal VEP in MS the technique is certainly capable of revealing abnormalities in the visual system which, by inference, are the result of clinically undetectable lesions. The correlation of these abnormalities with MS diagnosed as definite on other grounds supports the validity of the concept. It should be emphasised again that the observed values for latency depend upon the technique used, and this, as well as factors of patient selection, may affect the results obtained. Finally, the value of the technique in early diagnosis of MS must depend on more thorough follow-up studies than have so far been reported.

We are grateful to our colleagues who referred patients for investigation. This work was supported in part by the Medical Research Council.

\section{References}

Asselman, P., Chadwick, D. W., and Marsden, C. D. (1975). Visual evoked responses in the diagnosis and management of patients suspected of multiple sclerosis. Brain, 98, 261-282.

Halliday, A. M., McDonald, W. I., and Mushin, J. (1972). Delayed visual evoked response in optic neuritis. Lancet, 1, 982-985.

Halliday, A. M., McDonald, W. I., and Mushin, J. (1973). Visual evoked response in cliagnosis of multiple sclerosis. British Medical Journal, 4, 661664.

Halliday, A. M., McDonald, W. I., and Mushin, J. (1974). Delayed visual evoked responses in progressive spastic paraplegia. Electroencephalography and Clinical Neurophysiology, 37, 328.

Marshall, J. (1955). Spastic paraplegia of middle age. A clinico-pathological study. Lancet, 1, 693-695.

Mastaglia, F. L., Black, J. L., and Collins, D. W. K. (1976). Visual and spinal evoked potentials in diagnosis of multiple sclerosis. British Medical Journal, 3, 732.

McAlpine, D., Lumsden, C. E., and Acheson, E. D. (1972). Multiple Sclerosis. A Reappraisal. Churchill Livingstone: Edinburgh and London. 